\title{
Synthesis of Photocatalytic Niobate Nanosheet/Polymer Composite Microgel Particles through Microfluidic Approach
}

\author{
Yumeng Ye ${ }^{1,2, a}$, Masanari Nishi,b, Yang Wenqiac, \\ Masahiro Takinoue ${ }^{3, \mathrm{~d}}$ and Nobuyoshi Miyamoto ${ }^{2, \mathrm{e}^{*}}$ \\ ${ }^{1}$ Key Laboratory of Soft Chemistry and Functional Materials, Nanjing University of Science and \\ Technology, Ministry of Education, Nanjing 210094, China \\ ${ }^{2}$ Department of Life, Environment and Materials Science, Fukuoka Institute of Technology, Fukuoka \\ 811-0295, Japan \\ ${ }^{3}$ Department of Computer Science, Tokyo Institute of Technology, 4259 Nagatsuta-cho, Midori-ku, \\ Yokohama 226-8502, Japan \\ ambm17105@bene.fit.ac.jp, ${ }^{\mathrm{b}} \mathrm{mbm17102@bene.fit.ac.jp,}{ }^{\mathrm{b}} \mathrm{mbm} 18201 @$ bene.fit.ac.jp, \\ dtakinoue@c.titech.ac.jp, emiyamoto@fit.ac.jp
} Keywords: microfluidic, poly $(N, N$-dimethylacrylamide $)$ microgels, composite microgels,
nanosheets, photocatalyst

Abstract. Polydimethylacrylamide (PDMA) microgels and niobate nanosheet/PDMA composite microgels were fabricated by using a microfluidic device. Morphologies and sizes of the composite microgels were tuned by adjusting synthetic conditions such as viscosity of oil phases, hydrophilicity and concentration of surfactants, and flow rates of oil phase and water phase. Furthermore, it was found that the dispersion of nanosheets was better when the composite microgels were synthesized by photopolymerization compared to redox polymerization.

\section{Introduction}

Microgels are micrometer-sized hydrogel particles containing a cross-linked polymer network [1] that are swollen by solvent, usually water. Due to its unique features, microgels can be used as carriers for various kinds of functional materials, including drugs, cells, proteins and catalysts [2, 3, 4]. Such soft capsules are attractive candidates for both basic research and practical applications [5], e.g., drug delivery system, sensor and colloidal crystals $[6,7,8]$.

Recently, a new approach, microfluidic technology [9], to fabricate microgels has attracted considerable attention. In this method, a continuous fluid is cut off and emulsified to generate micrometer-sized droplet precursors inside a microfluidic device. The mechanism of droplets formation is related to the balance of shear stress and interfacial tension between two or more incompatible fluids [10]. Usually, an aqueous solution is the dispersed phase, which is emulsified to form water-in-oil droplets by an oil solution. Subsequently, these droplets containing monomers transform into microgel particles through polymerization and cross-linking. Compared with traditional synthetic methods, such as solution polymerization and emulsion polymerization, fabricating microgels through microfluidic approach is advantageous to prepare uniform microgels with precise control over their size, shape and monodispersity [1], resulting in a great potential for industrial production.

Meanwhile, hexaniobate $\mathrm{K}_{4} \mathrm{Nb}_{6} \mathrm{O}_{17}$ is a typical lamellar semiconductor material, which is composed of stacked asymmetrical $\mathrm{Nb}_{6} \mathrm{O}_{17}{ }^{4-}$ layers [11, 12]. $\mathrm{K}_{4} \mathrm{Nb}_{6} \mathrm{O}_{17}$ possess remarkable photocatalytic activity $[11,13,14]$ and the photocatalytic performance is further enhanced by exfoliating the bulk $\mathrm{K}_{4} \mathrm{Nb}_{6} \mathrm{O}_{17}$ crystals into $2 \mathrm{D}$ nanosheets $[15,16,17,18]$ because effective surface area drastically increases by the exfoliation. However, the niobate nanosheets aggregate easily in the presence of salt etc., resulting in decreased catalytic activity. Even if the aggregation is prevented, collecting the nanometer-sized catalyst particles for reuse is problematic. Thus, we need to overcome these limitations to use the nanosheet catalyst for wide industrial applications. 
In this study, to solve these problems, immobilization of niobate nanosheets in polymer microgels was investigated through a microfluidic approach. Although microfluidic approach for microgel synthesis is well-established by many researchers, this method is applied for nanosheet/polymer composite system for the first time. Because microfluidic synthesis is affected by many factors such as viscosity of the oil and water phases, application of this method to the anisotropic colloidal system of nanosheets was not straightforward. We explored the synthetic conditions to obtain microgels with desired shapes, sizes as well as good dispersion of the nanosheets.

\section{Experimental}

Materials. KF-96L-1Cs and KF-6028 were purchased from Shin-Etsu Chemical Co., Ltd. The other reagents were purchased from Tokyo Chemical Industry Co., Ltd. All reagents were used as received. Instrumentation. Composite microgels were observed by a polarizing microscope (Olympus, BX-51).

Manufacture of Microfluidic Device. Microchannels with depth and width of $0.5 \mathrm{~mm}$ inside the device were designed by Auto CAD. Then the microchannels were carved on a polymethyl methacrylate (PMMA) plate by a computer numerical control machine tool (Roland DG, ZBX-650). After washing, the PMMA plate was pressed with a same blank plate for $20 \mathrm{~min}$ at $90{ }^{\circ} \mathrm{C}$ by a hot press (AS ONE, AH-2003), making the channels sealed inside the device. At last, three plastic capillaries with an inner diameter of $0.5 \mathrm{~mm}$ were bonded to two inlets and an outlet, respectively.

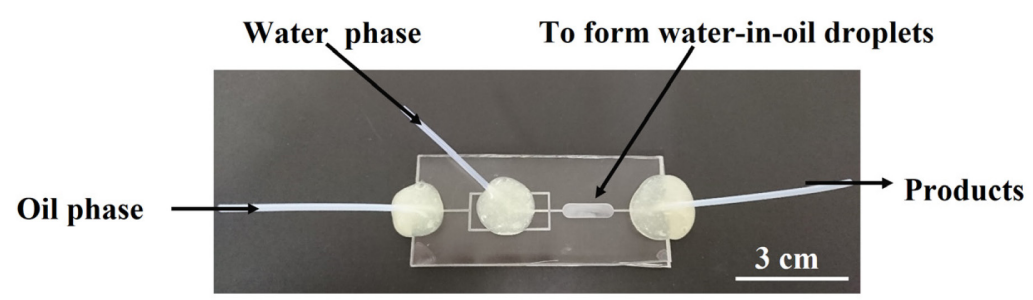

Fig. 1. Photograph of the microfluidic device.

Fig. 1 is a photograph of the obtained microfluidic device and flow directions of fluids are indicated by the arrows. The water-in-oil droplets are generated in the hollow part of the device, then the products were collected through the capillary on the right side of the device.

Preparation of PDMA Microgels without Nanosheets. Five kinds of oil phases were prepared: (a) $5 \mathrm{wt} \% \mathrm{KF}-6028$ (surfactant) and $1 \mathrm{wt} \% N, N, N^{\prime}, N^{\prime}$-tetramethylethylenediamine (TEMED, reaction accelerator) were dissolved in KF-96L-1Cs (a type of silicone oil); (b) $5 \mathrm{wt} \%$ Span 80 (surfactant) and $1 \mathrm{wt} \%$ TEMED were dissolved in KF-96L-1Cs; (c) Span 80 with concentrations of $0 \mathrm{wt} \%, 3 \mathrm{wt}$ $\%$ or $5 \mathrm{wt} \%$ as well as $1 \mathrm{wt} \%$ TEMED were dissolved in mineral oil. These oil phases were stirred thoroughly and stored at room temperature. As the water phase, $11.54 \mathrm{wt} \%$ monomer ( $N, N$-dimethylacrylamide, DMA $), 0.17 \mathrm{wt} \%$ cross-linker $(N, N$-methylenebisacrylamide, MBA) and $0.80 \mathrm{wt} \%$ redox initiator (ammonium persulfate, APS) were dissolved in DI water. The molar ratio of monomer to cross-linker is 100:1. Incubation solution [19] consisted of $1 \mathrm{wt} \%$ APS and $5 \mathrm{wt} \%$ TEMED in DI water. Both the water phase and incubation solution were stirred thoroughly and purged with nitrogen for $15 \mathrm{~min}$ and stored in a refrigerator.

During the experiment, flow rates of the oil phase and the water phase (listed in Table 1) were adjusted precisely by employing two syringe pumps (YMC, YSP-201). A phase contrast optical microscope (Nikon, ECLIPSE Ti) was used to observe the formation process of droplets in the device simultaneously. The products were collected in a vial containing some incubation solution when the emulsion became steady. The collected precursors were stored in the incubation solution for $12 \mathrm{~h}$, making the monomers polymerize sufficiently to obtain microgel particles. Finally, the upper oil phase was removed using a separating funnel. Then the microgel particles were rinsed with DI water several times and stored in DI water. 
Using oil phase containing $5 \mathrm{wt} \%$ Span 80 and 1 wt \% TEMED in mineral oil, the microgel sample prepared at flow rates of $120 \mu \mathrm{L} \mathrm{min}{ }^{-1}$ of the oil phase and $20 \mu \mathrm{L} \mathrm{min}^{-1}$ of the water phase is marked as Blank Gel.

Preparation of Nanosheet/PDMA Composite Microgels. Niobate nanosheets were obtained according to a previous report [20] as follows. First, layered hexaniobate crystals were synthesized by a solid state reaction from a mixture of niobium pentoxide and potassium carbonate powders. Then, the layered crystals were exfoliated into nanosheets by treatment in an aqueous solution of propylammonium chloride. The nanosheet colloid was freeze-dried to be used in the next step.

For redox polymerization, the oil phase containing $5 \mathrm{wt} \%$ of Span 80 and $1 \mathrm{wt} \%$ of TEMED in mineral oil was prepared. $0.5 \mathrm{wt} \%$ of freeze-dried nanosheet powder was added to the water phase solution used for the synthesis of pure PDMA microgels. Then the composite microgels (marked as R-Composite Gel) were prepared by using the microfluidic device at the flow rates of $120 \mu \mathrm{L} \mathrm{min}^{-1}$ of the oil phase and $20 \mu \mathrm{L} \mathrm{min}{ }^{-1}$ of the water phase.

For photopolymerization, TEMED, APS or the incubation solution were not used because the niobate nanosheets act as photoinitiators [21]. The collected droplets were irradiated by a high-pressure mercury lamp (USHIO, SX-UI501HQ) for $15 \mathrm{~min}$ to form microgels. The other synthetic conditions were the same as that of R-Composite Gel. The sample is marked as P-Composite Gel.

\section{Results and Discussion}

Preparation of Microgels. The mechanism for preparation of microgels in the microfluidic device by redox polymerization is described in Fig. 2. The oil phase and water phase were pumped into the device from the inlets B and A, respectively, in the direction of the arrows. At the intersection of two phases, owing to the shear stress of oil phase acting on the water phase as well as emulsification of surfactants in the oil phase, the water phase was dispersed as a microscale water-in-oil droplet, in which the monomer, cross-linker and initiator are contained. Meanwhile, TEMED dissolved in the oil phase diffused into the droplets to accelerate polymerization of DMA monomers [19].

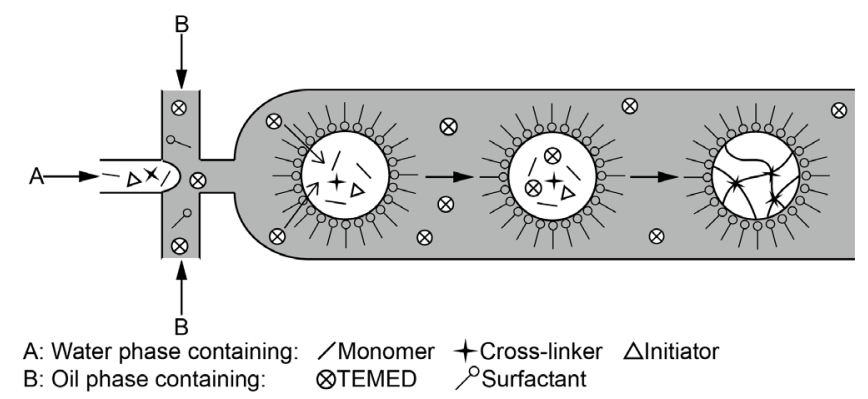

Fig. 2. Schematic illustration for fabrication of microgels in the microfluidic device.

Microgel precursors were collected in an incubation solution containing a high concentration of TEMED and APS to obtain cross-linked microgels. As shown in Fig. 3, collected precursors gathered at the bottom of the oil phase. After polymerization and cross-linking process of the precursors, the particles gradually sank into the incubation solution and swelled and precipitated at the bottom of the collection vial, finally. After separation and rinse, the microgels were transferred to DI water. Compared with direct collection method, the employment of the incubation solution is advantageous to accelerate the gelation process and facilitate the removal of the oil phase. Besides, agglomeration between precursors can also be avoided. 


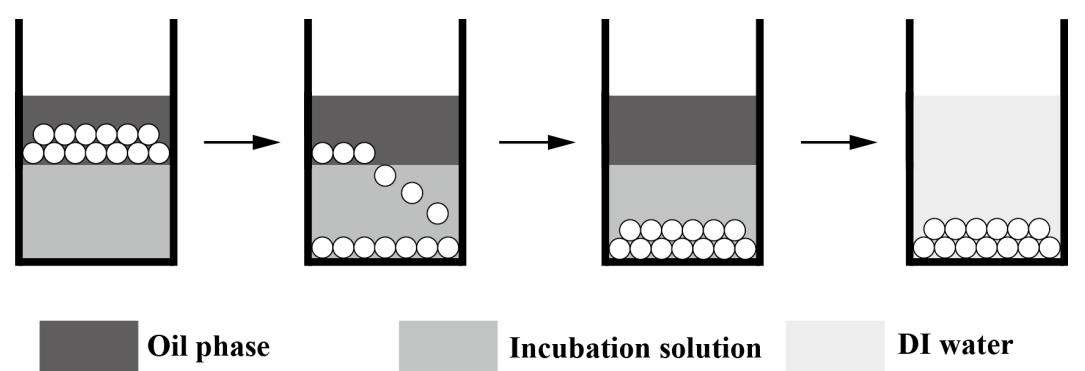

Fig. 3. Schematic illustration for incubation and precipitation of microgels.

Morphologies and sizes of the microgels were tunable by changing the kinds of surfactant and oil. As shown in Fig. 4 (a), (b) and (c), the shape of the microgels ranged from ellipsoid to sphere, and the particle size was also different. We infer that the ellipsoidal gel in Fig. 4 (a) was formed by a squeeze of collecting capillary because the diameter of droplet precursors was larger than that of collecting capillary $(500 \mu \mathrm{m})$, while the particles in Fig. 4 (b) were squeezed a little, indicating corresponding precursors' size was smaller. The used surfactants were KF-6028 and Span 80, and their hydrophile-lipophile balance (HLB) numbers are 4.0 and 4.5 respectively. The larger HLB number displays stronger hydrophilicity, leading to the easier generation of water-in-oil droplets and the smaller size of droplets in the microfluidic device.

Meanwhile, microgels in Fig. 4 (c) had a spherical shape with good monodispersity, and diameters were around $600 \mu \mathrm{m}$. As we evaluated the sizes of the gel particles by observing 65 particles one by one on the microscopy, the average size was evaluated as $572 \mu \mathrm{m}$ with the standard deviation of $37 \mu \mathrm{m}$, that is $6 \%$ of the average value. The difference of sample (b) from (c) was caused by the type of oil phases: low viscosity silicone oil (KF-96L-1Cs) and mineral oil for (b) and (c), respectively. The kinematic viscosity of silicone oil and mineral oil are $1 \mathrm{~mm}^{2} \mathrm{~s}^{-1}$ and $15 \mathrm{~mm}^{2} \mathrm{~s}^{-1}$ at room temperature. The larger viscosity of the oil phase leads to the stronger shear stress on the water phase, resulting in the smaller diameter of the droplet precursors.

Microgel diameter is also influenced by surfactant concentration in the oil phase. Mineral oil without surfactant, as well as mineral oil with $3 \mathrm{wt} \%$ and $5 \mathrm{wt} \%$ of Span 80 , were utilized to fabricate microgels, respectively. The microgel was not obtained without the surfactant, because the emulsified droplets combined with each other immediately. As illustrated in Fig. 4 (c) and (d), it is found that the diameter of microgels decreased from $800 \mu \mathrm{m}$ to $600 \mu \mathrm{m}$ with the increase of surfactant concentration from $3 \mathrm{wt} \%$ to $5 \mathrm{wt} \%$. The higher concentration of surfactant possesses the stronger emulsification capability on the water phase, resulting in the smaller droplet diameters.

Furthermore, microgel size is also tunable by adjusting the flow rates of oil phase and water phase. According to Fig. 4 (c), (e) and (f), the gradual shrinkage of microgels could be observed with the increase of flow rate of oil phase relative to the water phase, which is owing to the enlargement of shear stress acting on the water phase. 

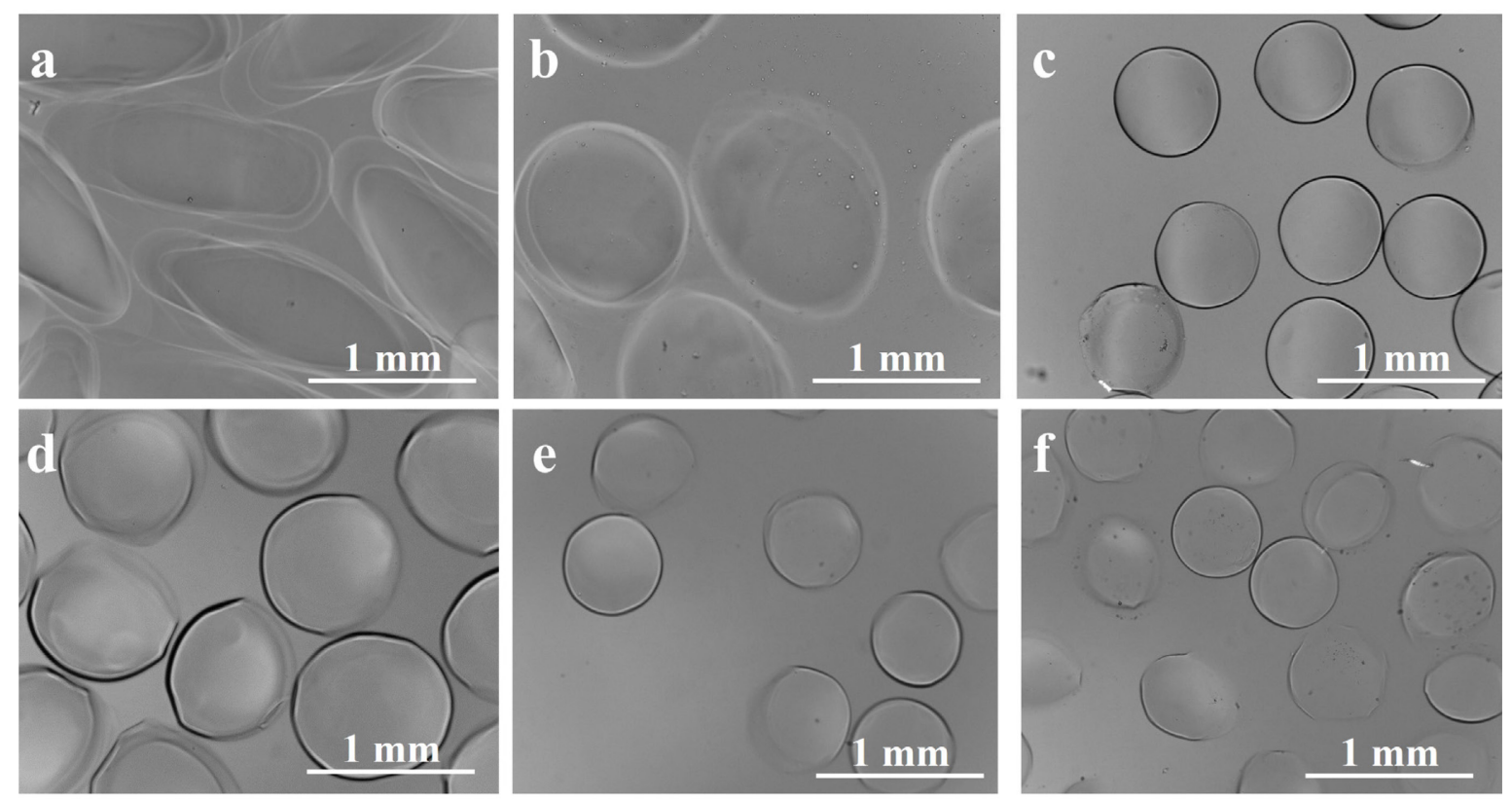

Fig. 4. Optical microscopy images of the microgel particles synthesized under various conditions. Images (a), (b), (c) are the samples prepared with different kinds of surfactants and oil phases: (a) silicone oil and KF-6028, (b) silicone oil and Span 80, (c) mineral oil and Span 80. Images (c), (d) compares the samples prepared from different concentrations of Span 80: (c) $3 \mathrm{wt} \%$, (d) $5 \mathrm{wt} \%$. Images (c), (e), (f) compares the samples prepared from different flow rates of oil phase and water phase: (c) oil phase: $120 \mu \mathrm{L} \mathrm{min}{ }^{-1}$, water phase: $20 \mu \mathrm{L} \mathrm{min}^{-1}$, (e) oil phase: $120 \mu \mathrm{L} \mathrm{min}^{-1}$, water phase:

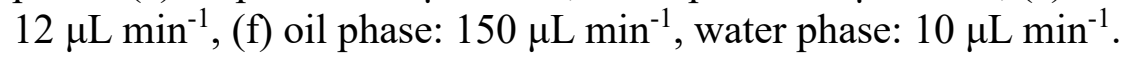

In addition, the molar ratio of monomer and cross-linker is also an important parameter. The droplet precursors ruptured during the incubation process when the amount of cross-linker is too little (molar ratio of DMA to BIS is 1000: 1). Meanwhile, excessive cross-linking occurred if there is too much cross-linker (molar ratio of DMA to BIS is 2: 1), which resulted in white polymer particles rather than microgels containing massive water. Besides, the concentration of TEMED in the oil phase should not be too high. Otherwise, the microchannel will be blocked due to rapid polymerization reaction at the intersection of the microfluidic device.

As summarized in Table 1, we concluded that the size of microgels decreases with the increase of viscosity of oil phase, the increase of HLB number and concentration of surfactant, as well as the increase of flow rate of oil phase relative to water phase within a certain range.

Table 1. Synthetic conditions for microgel particles.

\begin{tabular}{ccccccc}
\hline $\begin{array}{c}\text { Synthetic } \\
\text { condition }\end{array}$ & Oil & Surfactant & $\begin{array}{c}\text { Content of } \\
\text { surfactant } \\
{[\mathrm{wt} \%]}\end{array}$ & $\begin{array}{c}\text { Flow rate of } \\
\text { oil phase } \\
{\left[\mu \mathrm{L} \mathrm{min} \mathrm{min}^{-1}\right]}\end{array}$ & $\begin{array}{c}\text { Flow rate of } \\
\text { water phase } \\
{\left[\mu \mathrm{L} \mathrm{min}{ }^{-1}\right]}\end{array}$ & $\begin{array}{c}\text { Diameter of } \\
\text { microgels } \\
{[\mu \mathrm{m}]}\end{array}$ \\
\hline A & KF-96L-1Cs & KF-6028 & 5 & 120 & 20 & $>1000$ \\
B & KF-96L-1Cs & Span 80 & 5 & 120 & 20 & 1000 \\
C $^{*}$ & Mineral oil & Span 80 & 5 & 120 & 20 & 600 \\
D & Mineral oil & Span 80 & 3 & 120 & 20 & 800 \\
E & Mineral oil & Span 80 & 5 & 120 & 12 & 500 \\
F & Mineral oil & Span 80 & 5 & 150 & 10 & 400 \\
\hline
\end{tabular}

* R-Composite Gel and P-Composite Gel were synthesized with this condition. 
Preparation of Composite Microgels. As shown in Fig. 5, Blank Gel, R-Composite Gel and P-Composite Gel were observed by the polarizing microscope under crossed polarizers. Obviously, the two kinds of composite microgels are of spherical shape with diameters of $600 \mu \mathrm{m}$, which is consistent with the size of PDMA microgels prepared under the same conditions. It is demonstrated that the spherical shape and size of microgels were maintained well even if niobate nanosheets were loaded.
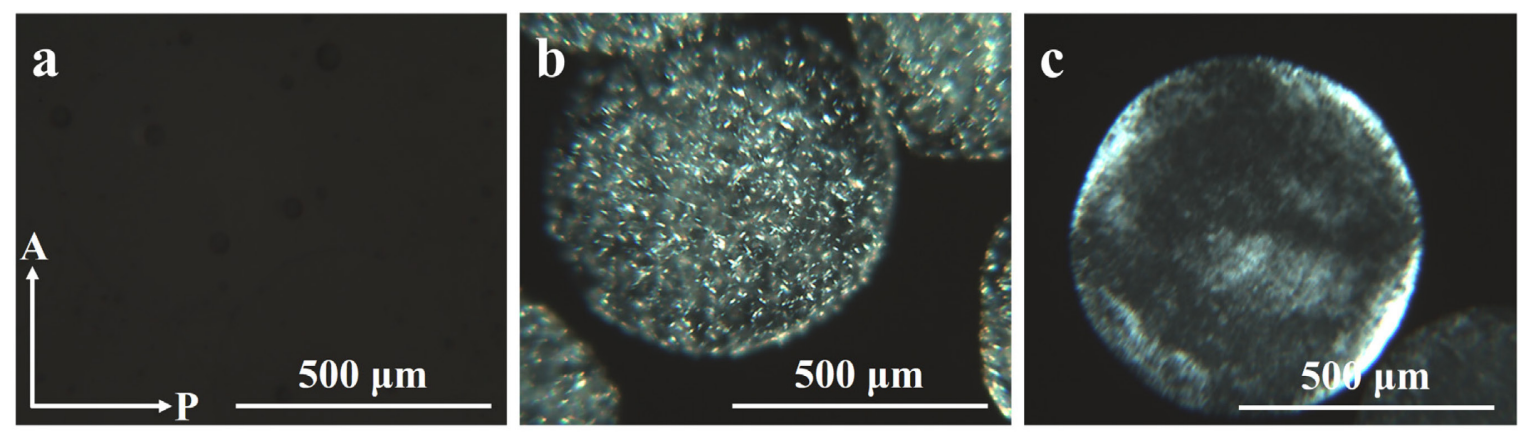

Fig. 5. Crossed polarizing microscopy images of (a) PDMA microgel without nanosheets, (b) R-Composite Gel and (c) P-Composite Gel.

Dispersion state of niobate nanosheets in the composite microgels is further studied from the microscopic images. PDMA microgels without nanosheets showed a totally dark image (Fig. 5a), indicating that the polymer network inside the microgel is isotropic without any birefringence. In the R-Composite Gel (Fig. 5b), lots of bright spots of around $10 \mu \mathrm{m}$ are observed. These spots represent birefringent layered niobate crystals that were formed by assembly and restacking of the nanosheets during the synthetic process. We suppose that the use of the ionic initiator APS caused the restacking of niobate nanosheets during the redox polymerization. It is generally known that repulsive interaction between charged colloidal particles is reduced in the presence of ions, especially multivalent ones, to cause coagulation of the particles[22]. Because we used $0.80 \mathrm{wt} \%(0.036 \mathrm{M})$ of the initiator APS, the ionic strength is calculated as $0.21 \mathrm{M}$ and this could be high enough for the nanosheets to be coagulated and restacked.

In contrast, in P-Composite Gel (Fig. 5c), vague birefringent domains with the size of several hundred microns were observed, which is similar to those observed in the lyotropic liquid crystal phase of nanosheets colloids [23]. The strong birefringence is found at the diagonal edges of the spherical microgel (the edges at the angle of 45 degrees to the polarizer/analyzer directions), indicating that the liquid crystalline nanosheets are aligned along the water/oil interface. It is plausible that part of the nanosheets are adsorbed on the water/oil interface of the water-in-oil droplet, inducing the alignment of the nanosheets liquid crystal inside the droplets. It was reported that nanosheets tend to adsorb on the interface and act as an emulsifying agent to form stable pickering emulsion[24]. Although the surfactant used here, Span80, is not a non-ionic one and the interaction with nanosheet is not very strong, the adsorption is possible. It is notable, in general, that the liquid crystalline behavior of the nanosheets only appears when most of the nanosheets are dispersed as single layers. Our observation of liquid crystalline behavior in the P-Composite Gel indicates that the nanosheets are dispersed as monolayers, which is preferable for the application as catalysts. Thus, the niobate nanosheets were successfully immobilized in both R-Composite Gel and P-Composite Gel, while the degree of exfoliation was much better in the P-Composite Gel than R-Composite Gel.

\section{Conclusion}

PDMA microgels and niobate nanosheet/PDMA composite microgels were synthesized successfully through the microfluidic device with the precise control of the sizes, shapes, and dispersion of the embedded nanosheets. We found that the size of microgel particles decreases with the increase of viscosity of oil phase, the increase of HLB number and concentration of surfactant, as 
well as the increase of flow rates of oil phase relative to water phase within a certain range. The dispersion of the nanosheets in the composite microgels prepared by photopolymerization was superior to those prepared by redox polymerization. Taking advantage of the photocatalytic activity and large specific surface area of the niobate nanosheets as well as high permeability of polymer gel, the present composite microgels will be applicable as novel photocatalysts for organic synthesis or water splitting for hydrogen production because the aggregation of the nanosheets during catalytic reaction is expected to be prevented and recycling of the catalysts after use will be much easier compared to nanosheets alone.

\section{Acknowledgment}

This research was partly supported by: KAKENHI (\#15K05657, \#15KK0217, and \#18K19135); Research Center for Materials and Energy Devices of Fukuoka Institute of Technology (FIT-ME) funded by Strategic Research Foundation Grant-Aided Project for Private University from MEXT; and Electronics Research Laboratory of Fukuoka Institute of Technology.

\section{References}

[1] Seiffert, S., Small but smart: sensitive microgel capsules. Angew. Chem. 52 (2013) 11462-11468.

[2] Hsu M N, Luo R, Kwek K Z, et al. Sustained release of hydrophobic drugs by the microfluidic assembly of multistage microgel/poly (lactic-co-glycolic acid) nanoparticle composites. Biomicrofluidics. 9 (2015) 052601.

[3] Panda P, Ali S, Lo E, et al. Stop-flow lithography to generate cell-laden microgel particles. Lab Chip. 8 (2008) 1056-1061.

[4] Lu Y, Proch S, Schrinner M, et al. Thermosensitive core-shell microgel as a "nanoreactor" for catalytic active metal nanoparticles. J. Mater. Chem. 19 (2009) 3955-3961.

[5] Tumarkin, E., Kumacheva, E., Microfluidic generation of microgels from synthetic and natural polymers. Chem. Soc. Rev. 38 (2009) 2161-2168.

[6] Hu Y, Wang Q, Wang J, et al. Shape controllable microgel particles prepared by microfluidic combining external ionic crosslinking. Biomicrofluidics. 6 (2012) 026502.

[7] Chu L Y, Utada A S, Shah R K, et al. Controllable monodisperse multiple emulsions. Angew. Chem. 46 (2007) 8970-8974.

[8] Kanai T, Lee D, Shum H C, et al. Fabrication of tunable spherical colloidal crystals immobilized in soft hydrogels. Small. 6 (2010) 807-810.

[9] Manz A, Graber N, Widmer H M. Miniaturized total chemical analysis systems: a novel concept for chemical sensing. Sens. Actuator B-Chem. 1 (1990) 244-248.

[10] Shah R K, Kim J W, Agresti J J, et al. Fabrication of monodisperse thermosensitive microgels and gel capsules in microfluidic devices. Soft Matter. 4 (2008) 2303-2309.

[11] Zhong T, Tang J, Zhu M, et al. Synthesis and characterization of layered niobate $\mathrm{K}_{4} \mathrm{Nb}_{6} \mathrm{O}_{17}$ thin films by niobium-chelated precursor. J. Cryst. Growth. 285 (2005) 201-207.

[12] Gasperin M, Le Bihan M T. Mecanisme d'hydratation des niobates alcalins lamellaires de formule $\mathrm{A}_{4} \mathrm{Nb}_{4} \mathrm{O}_{17}(\mathrm{~A}=\mathrm{K}, \mathrm{Rb}, \mathrm{Cs})$. J. Solid State Chem. 43 (1982) 346-353.

[13] Domen K, Kudo A, Shinozaki A, et al. Photodecomposition of water and hydrogen evolution from aqueous methanol solution over novel niobate photocatalysts. J. Chem. Soc., Chem. Commun. 4 (1986) 356-357. 
[14] Kudo A, Tanaka A, Domen K, et al. Photocatalytic decomposition of water over $\mathrm{NiO}-\mathrm{K}_{4} \mathrm{Nb}_{6} \mathrm{O}_{17}$ catalyst., J. Catal. 111 (1988) 67-76.

[15] Sarahan M C, Carroll E C, Allen M, et al. $\mathrm{K}_{4} \mathrm{Nb}_{6} \mathrm{O}_{17}$-derived photocatalysts for hydrogen evolution from water: nanoscrolls versus nanosheets. J. Solid State Chem. 181 (2008) 1678-1683.

[16] Kimura N, Kato Y, Suzuki R, et al. Single-and double-layered organically modified nanosheets by selective interlayer grafting and exfoliation of layered potassium hexaniobate. Langmuir. 30 (2014) 1169-1175.

[17] Nakato T, Fujita T, Mouri E. Synergistic photocatalytic hydrogen evolution over oxide nanosheets combined with photochemically inert additives. Phys. Chem. Chem. Phys. 17 (2015) 5547-5550.

[18] Nakato T, Terada S, Ishiku T, et al. Photoinduced electron transfer in semiconductor-clay binary nanosheet colloids controlled by clay particles as a turnout switch. Appl. Catal. B-Environ. 241 (2019) 499-505.

[19] Raz N, Li J K, Fiddes L K, et al. Microgels with an interpenetrating network structure as a model system for cell studies. Macromolecules. 43 (2010) 7277-7281.

[20] Miyamoto N, Yamada Y, Koizumi S, et al. Extremely stable photoinduced charge separation in a colloidal system composed of semiconducting niobate and clay nanosheets. Angew. Chem. 119 (2007) 4201-4205.

[21] Sun J, Schmidt B V K J, Wang X, et al. Self-Standing Carbon Nitride-Based Hydrogels with High Photocatalytic Activity. ACS Appl. Mater. Interfaces. 9 (2017) 2029-2034.

[22] Miyamoto N, Ohsedo Y, Nakato T. "Chapter 8: Colloidal Nanosheets" in Inorganic Nanosheets and Nanosheet-Based Materials, eds Nakato T, Kawamata J, Takagi S.), 201-260 (Springer Japan, 2017).

[23] Miyamoto N, Nakato T. Liquid crystalline nanosheet colloids with controlled particle size obtained by exfoliating single crystal of layered niobate $\mathrm{K}_{4} \mathrm{Nb}_{6} \mathrm{O}_{17}$. J. Phys. Chem. B. 108 (2004) 6152-6159.

[24] Nakato T, Ueda H, Hashimoto S, Terao R, Kameyama M, Mouri E., "Pickering Emulsions Prepared by Layered Niobate $\mathrm{K}_{4} \mathrm{Nb}_{6} \mathrm{O}_{17}$ Intercalated with Organic Cations and Photocatalytic Dye Decomposition in the Emulsions" ACS Appl Mater Interfaces 108 (2012) 4338-4347. 